\title{
Coexistence of plasma cell neoplasia and myelodysplastic syndrome with excess blasts: case reports and literature review
}

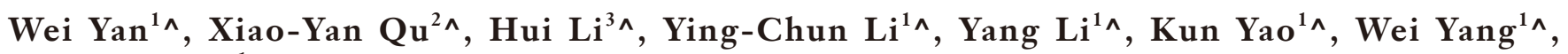 \\ Hui-Han Wang ${ }^{1 \wedge}$ \\ ${ }^{1}$ Department of Hematology, Shengjing Hospital of China Medical University, Shenyang, China; ${ }^{2}$ Department of Hematology, Jiangsu Province \\ Hospital, Nanjing, China; ${ }^{3}$ Department of Hematology, Sichuan Provincial People's Hospital, University of Electronic Science and Technology of \\ China, Chengdu, China \\ Contributions: (I) Conception and design: W Yan, HH Wang; (II) Administrative support: None; (III) Provision of study materials or patients: None; \\ (IV) Collection and assembly of data: W Yan, K Yao, YC Li, Y Li; (V) Data analysis and interpretation: W Yan; (VI) Manuscript writing: All authors; \\ (VII) Final approval of manuscript: All authors. \\ Correspondence to: Hui-Han Wang. Department of Hematology, Shengjing Hospital of China Medical University, 39 Huaxiang Road, Shenyang \\ 110022, China. Email: 240333079@qq.com.
}

Background The coexistence of plasma cell neoplasia such as multiple myeloma (MM) or monoclonal gammopathy of underdetermined significance (MGUS) with myeloid neoplasia such as myelodysplastic syndrome with excess blasts (MDS-EB) is exceedingly rare. Seeking to understand the clinical features of this dual hematological neoplasm and exploration of novel therapeutic approaches is warranted.

Methods: The cases of 7 patients diagnosed with both MGUS/MM coexisting with MDS-EB were reported. Moreover, this study reviewed and summarized 34 published cases of MDS including 7 cases of MDS-EB, describing the coexistence with plasma cell disease, and analyzed the clinical characteristics and survival of these cases.

Results: In total, 14 cases (7 reported here and 7 previously published) of MGUS/MM coexisting with MDS-EB were analyzed. Of these 14 patients, the median age was 65.5 years. Almost all (85.7\%) participants had severe anemia or pancytopenia, and nearly half $(42.9 \%)$ of the cases developed into acute myeloid leukemia (AML). Half of the participants showed osteolytic lesions. The median bone marrow plasma cell count was $23.0 \%$, and the median myeloid blast count was $7.5 \%$. Immunological analysis using flow cytometry confirmed the coexistence of 2 different clones, malignant myeloid clone (CD34 ${ }^{+}, \mathrm{CD}_{117^{+}}, \mathrm{HLA}^{-}$ $\mathrm{DR}^{+}, \mathrm{CD}_{33^{+}}$, and $\left.\mathrm{CD} 13^{+}\right)$and plasma clone $\left(\mathrm{CD} 38^{+}, \mathrm{CD} 138^{+}\right.$, and $\left.\mathrm{CD} 56^{+}\right)$. Patients with MGUS/MM and MDS-EB experience very poor therapeutic responses. A great number of patients (64\%) were reported to have no response or rapid relapse. The median overall survival (OS) was only 8 months for patients with MGUS/MM and MDS-EB, which was significantly shorter than that of those with MGUS/MM and MDSother type (median OS of 52 months) ( $\mathrm{P}=0.0009)$.

Conclusions: Herein, a type of malignant myeloid clone concurrent with plasma clone was reported, without previous exposure to chemotherapy, and poor prognosis of these patients was observed. However, standard treatment methods are still absent, which therefore heightens our awareness of this type of disease and the urgent need for further investigation to prolong survival.

\footnotetext{
^ ORCID: Wei Yan, 0000-0002-6305-1011; Xiao-Yan Qu, 0000-0003-0863-5417; Hui Li, 0000-0002-4550-7097; Ying-Chun Li, 00000002-0397-6776; Yang Li, 0000-0001-5519-2941; Kun Yao, 0000-0002-1947-7744; Wei Yang, 0000-0003-2945-2898; Hui-Han Wang, $0000-0003-1141-5263$.
} 


\begin{abstract}
Keywords: Myelodysplastic syndrome with excess of blasts (MDS-EB); multiple myeloma (MM); monoclonal gammopathy of underdetermined significance (MGUS); coexistence; prognosis
\end{abstract}

Submitted Oct 15, 2021. Accepted for publication Nov 18, 2021.

doi: 10.21037/apm-21-3210

View this article at: https://dx.doi.org/10.21037/apm-21-3210

\section{Introduction}

Myelodysplastic syndrome (MDS) and plasma cell malignancy are totally different clinical and pathologic entities in character. On the one hand, MDS primarily involves the bone marrow with variable degrees of cytopenia, morphological dysplasia, and increased blast count, and has a high risk of progression to acute myeloid leukemia (AML) (1). On the other hand, plasma cell disease is characterized by organ dysfunction, bone marrow associated with malignant plasma cells, and monoclonal gammopathy (2). Treatment for multiple myeloma $(M M)$, specifically with melphalan, lenalidomide, and so on, may increase the risk of developing secondary primary malignancies such as MDS $(3,4)$. Pemmaraju et al. analyzed 1,386 MM patients, and of these, 47 (3.4\%) were diagnosed with therapy-related myeloid neoplasm, while 34/47 (72.3\%) developed therapy-related MDS (5). Moreover, there is an inherently increasing risk of MDS in patients with plasma cell disorders who have no previous exposure to chemotherapy. Roeker et al. demonstrated that monoclonal gammopathy of underdetermined significance (MGUS) patients ( $\mathrm{n}=605)$ without chemotherapy exposure had a 2.4-fold risk of developing MDS compared to the healthy control cohort $(n=16,710)(6)$. These results suggest a high risk of MDS development in plasma disease regardless of chemotherapy exposure. Nonetheless, few cases presented the simultaneous occurrence of abnormal immature myeloid cells and plasma cells at first diagnosis, even without prior exposure to chemotherapy and long medical history (7-19). The underlying pathogenesis for this condition, and if the 2 diseases share a common genetic lesion remains to be determined. Recently, studies using whole exome sequencing on isolated bone marrow myeloid and plasma cells identified the 2 diseases as having likely emerged from separate clones (20). This piqued our awareness of this special type of disease and the urgent need for further investigation of its characteristics and exploration of effective therapy.

MDS with excess of blasts (MDS-EB) is a subtype of
MDS characterized by increased myeloid blasts and a high risk of progression to AML. The coexistence of MGUS/ MM with MDS-EB is less common than that of MGUS/ $M M$ and MDS. This uncommon clinical situation has complex features and complicated treatment, with unclear prognosis, and is difficult to diagnose early. To better understand this rare dual hematological neoplasm, we conducted case reports of patients from our hospital who were diagnosed with MDS-EB coexisting with MGUS/ MM. Moreover, we summarized the clinical characteristics and prognosis of MGUS/MM with MDS-EB through a literature review to provide some evidence for this specific group. We present the following article in accordance with the STROBE reporting checklist (available at https://dx.doi. org/10.21037/apm-21-3210).

\section{Methods}

Patients who had been newly diagnosed with plasma cell neoplasia (MGUS/MM) coexisting with MDS-EB between February 2015 and October 2020 were identified by searching the clinical databases of 3 institutions (Shengjing Hospital of China Medical University, Sichuan Provincial People's Hospital of University of Electronic Science and Technology of China, and Jiangsu Province Hospital). A total of 7 such patients were identified, for whom we subsequently compiled case reports. Meanwhile, we reviewed and summarized 34 cases of MDS including 7 MDS-EB published cases describing the coexistence with plasma cell disease entities combined with an analysis of the clinical characteristics and survival of these cases. The diagnosis of MGUS/MM and MDS-EB was based on morphology and immunophenotypic features according to the International MM Working Group criteria (21) and World Health Organization (WHO) classification for MDS (22), the secondary MDS caused by rheumatic system disease and tumor was excluded. The prognostic indicators of MDS and MM were monitored during the treatment, including bone marrow examination, blood routine, and $M$ protein. Given the rareness of this disease type, 
we searched for all previously published cases of plasma diseases coexisting with MDS in an effort to enhance our understanding of the features and prognosis of these cases. The clinical symptoms, laboratory data, diagnostic procedure, medical record, responses after treatment, and follow-ups were reported and summarized.

All procedures performed in this study involving human participants were in accordance with the Declaration of Helsinki (as revised in 2013). The study was approved by The Human Investigation Committee of China Medical University (No. 2020PS510K) and informed consent was taken from all the patients.

\section{Statistical analyses}

Survival curves of these groups were compared using the logarithmic rank test. At the same time, the univariate logarithmic rank test was used to examine the effect of other variables on survival. The Windows-specific version 5.0 of GraphPad Prism (GraphPad Software, San Diego, CA, USA) was used for image rendering.

\section{Results}

The 7 participants reported herein were diagnosed with MDS-EB-1 coexisting with MGUS/MM. To the best of our knowledge, this is the largest number of these types of cases reported together. The characteristics and clinical course of the 7 participants are shown in Table 1. Furthermore, our search resulted in the obtainment of another 34 published cases describing the coexistence of plasma cell disease with MDS (Table S1), among which 7 had been diagnosed with MGUS/MM coexisting with MDS-EB. A total of 14 patients were included for analysis of clinical features and survival (Table 2).

For the 14 patients with concurrent MGUS/MM and MDS-EB, the median age was 65.5 years (range, 54 to 79 years), which was younger overall than the MGUS/ MM-MDS patients (median age, 72.9 years; range, 47 to 92 years) (Table S1), and appearing younger than the MM patients (median age at onset, 71 years for men and 74 years for women) (23). Among the 14 patients, 10 were males, and 4 were females ( $M: F=2.5: 1)$. The majority $(85.7 \%$, $12 / 14$ ) of patients had severe anemia or pancytopenia, and $6(42.9 \%)$ cases developed into AML, demonstrating the existence of a myeloid clone. Half of the participants or $50 \%(7 / 14)$ had osteolytic lesions, which was lower than the published rate of osteolytic lesions at new diagnosis of MM
(70-80\%) (24). For the type of $M$ protein, 6 (42.9\%) were immunoglobulin $\mathrm{G}(\mathrm{IgG})$ type, 4 (28.6\%) IgA type, 1 (7.1\%) IgM type, and 3 (21.4\%) light chain type.

The morphology showed an increased percentage of simultaneously occurring myeloid blasts and atypical plasma cells. Bone marrow plasma cell percentage ranged from $2.4 \%$ to $66.5 \%$ (median, $23.0 \%$ ), and myeloid blast percentage ranged from $0.8 \%$ to $22.0 \%$ (median, $7.5 \%$ ). Immunological analysis by flow cytometry confirmed the coexistence of 2 different clones: malignant myeloid clone $\left(\mathrm{CD} 34^{+}, \mathrm{CD} 117^{+}, \mathrm{HLA}-\mathrm{DR}{ }^{+}, \mathrm{CD} 33^{+}\right.$and $\left.\mathrm{CD} 13^{+}\right)$and plasma clone $\left(\mathrm{CD} 38^{+}, \mathrm{CD} 138^{+}\right.$and $\left.\mathrm{CD} 56^{+}\right)$(Figure 1).

Patients diagnosed with MGUS/MM coexisting with MDS-EB displayed a very poor therapeutic response, regardless of targeting the myeloid clone (doxorubicin + cytarabine) or plasma clone (melphalan + prednisone, bortezomib + dexamethasone), and most of the patients $(64 \%, 9 / 14)$ reportedly had no response (Table 2). The combination of proteasome inhibitor (PI) and immunomodulators (IMiD) herein seemed to yield a favorable and rapid response. A total of 4 patients reached complete response and 1 achieved partial response after PTD (bortezomib, thalidomide, and dexamethasone), RVD (bortezomib, lenalidomide, and dexamethasone), or IRD (ixazomib, lenalidomide, and dexamethasone). Unfortunately, rapid relapse and progression of the disease were observed in these 4 patients.

The follow-up period was 3-12 months, and the median overall survival (OS) was 8 months for patients with MM/ MGUS coexisting with MDS-EB, the longest survival time was 15 months, while the median OS of patients diagnosed with MM/MGUS coexisting with MDS-non-EB type was 52 months.

The MDS-EB subtype of MDS has the characteristics of increased myeloid blast count in peripheral blood or bone marrow. It has more myeloid clones and a high risk for AML transformation. To compare the features of MGUS/MM presenting simultaneously with MDS-EB to MGUS/MM presenting simultaneously with other subtypes of MDS, all previously published cases of plasma diseases coexisting with MDS were summarized. We located a total of 34 such previously published patients. The main clinical and biological characteristics of 41 patients (34 published and 7 cases we reported here) are presented in Table S1. Among the 41 patients, $31.7 \%$ (13/41) were diagnosed with MGUS, $12.2 \%(5 / 41)$ with smoldering multiple myeloma (SMM), and $56.1 \%(23 / 41)$ with MM. There is some heterogeneity among the diseases categorized as MDS. Concerning the 
Table 1 The characteristics and clinical course in seven patients we reported here with coexistent MGUS/MM and MDS-EB-1

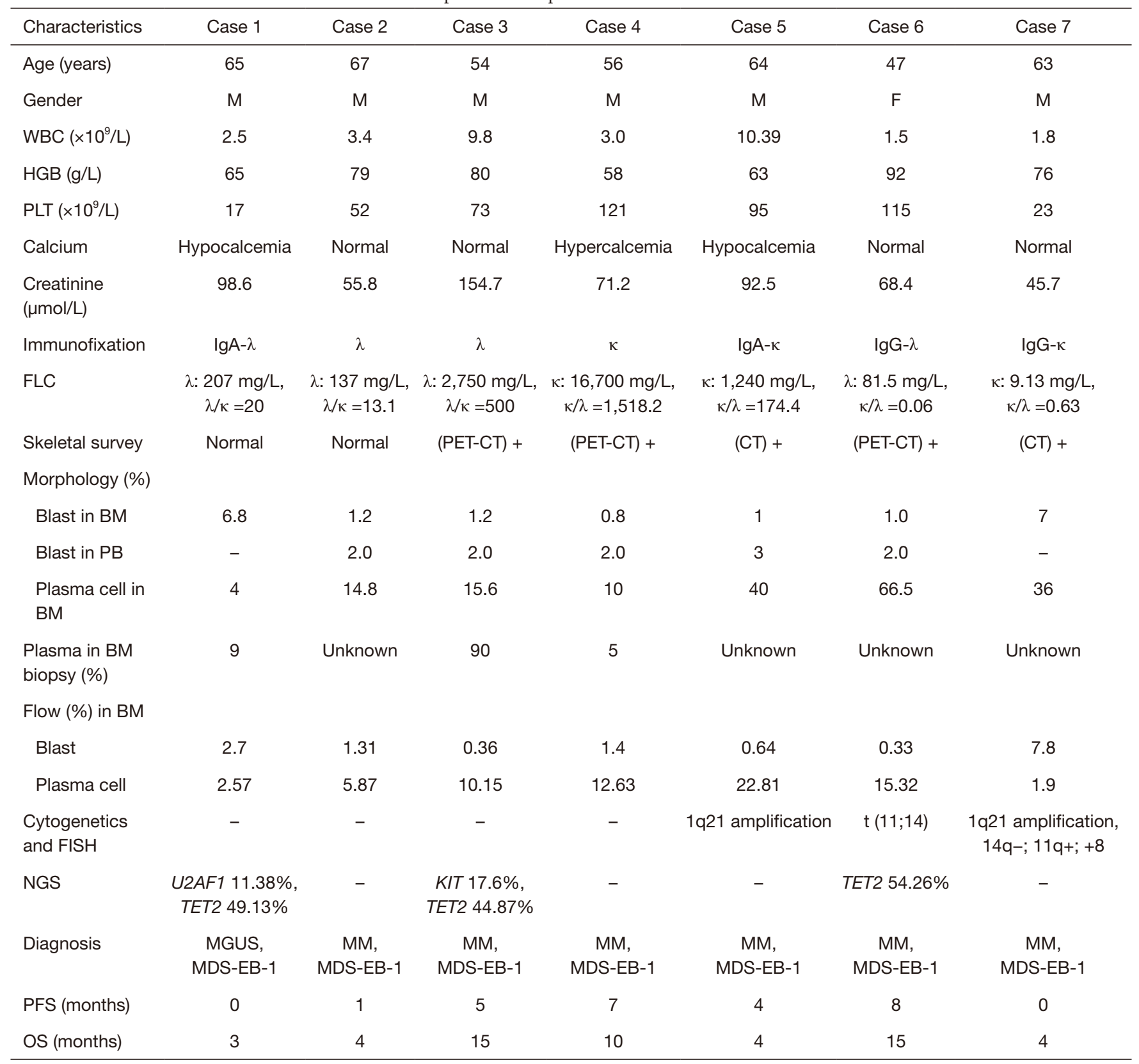

-: no significant change be detected; (PET-CT) +: PET-CT suggested bone destruction; (CT) +: CT suggested bone destruction. MGUS, monoclonal gammopathy of undetermined significance; MM, multiple myeloma; MDS-EB, myelodysplastic syndrome with excess blasts; WBC, white blood cell; HGB, hemoglobin; PLT, platelet; FLC, free light chain; BM, bone marrow; PB, peripheral blood; FISH, fluorescence in situ hybridization; PFS, progression free survival; OS, overall survival; PET, positron emission tomography; CT, computed tomography.

MDS classification, $34.1 \%(14 / 41)$ of cases were MDSEB, 29.3\% (12/41) were myelodysplastic syndromes with multilineage dysplasia (MDS-MLD), 12.2\% (5/41) were myelodysplastic syndromes with single lineage dysplasia (MDS-SLD), 12.2\% (5/41) were myelodysplastic syndromes with deletion of the long arm of chromosome 5 (MDS-5q-), $7.3 \%(3 / 41)$ were myelodysplastic syndromes unclassifiable (MDS-U), and 4.9\% (2/41) were myelodysplastic syndromes with ring sideroblasts (MDS-RS). A large proportion of cases were MDS-EB coexisting with plasma cell disease, and the 


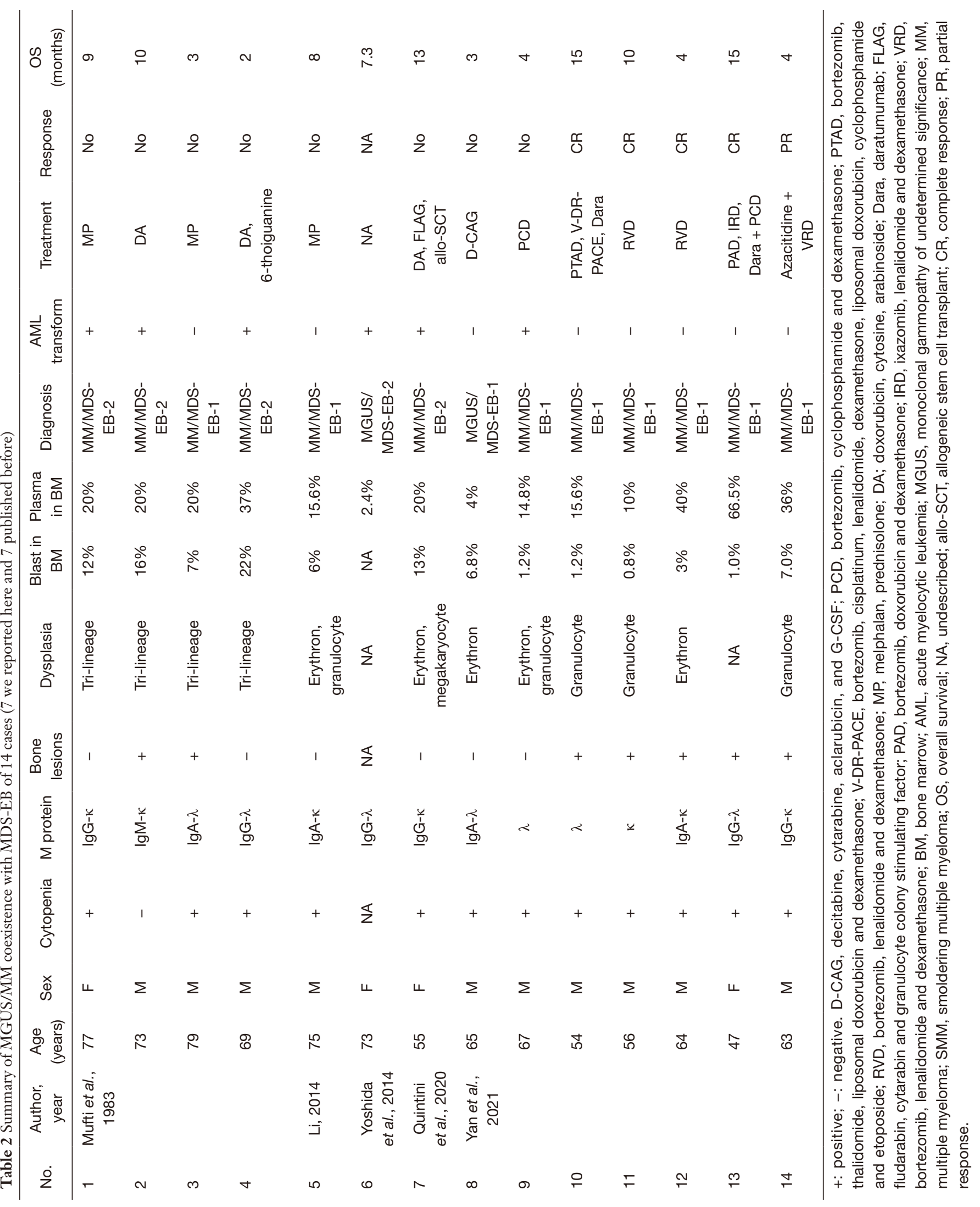


A

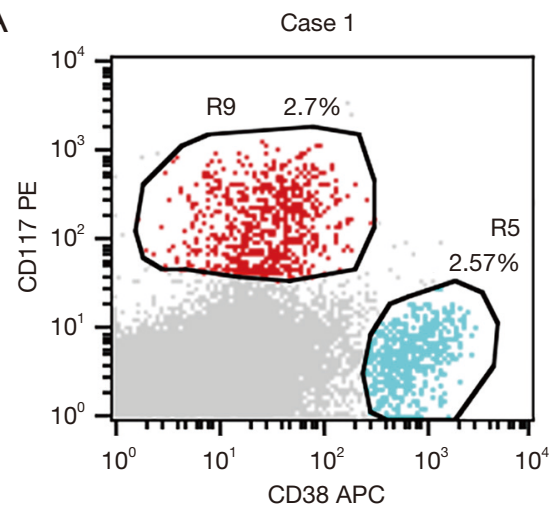

C

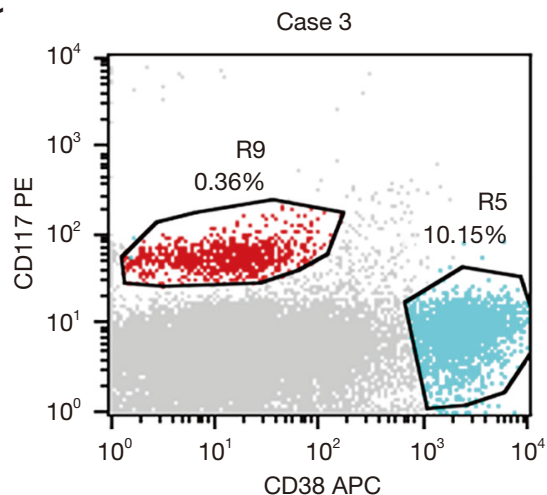

B

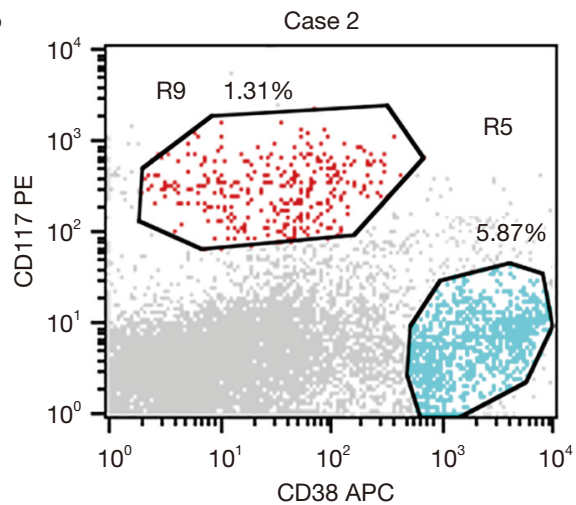

D

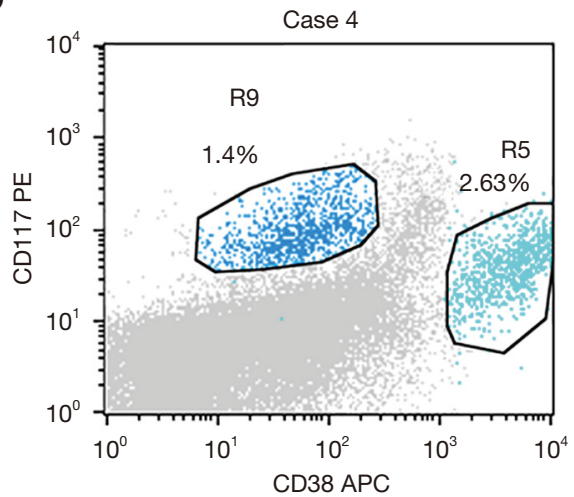

R5: plasma clone; R9: myeloid clone.

Figure 1 FACS plots to show the coexistence of CD117 positive monoclonal immature myeloid cells and CD38 positive clonal plasma cells in one patient. (A-D) indicates cases 1-4. R5: plasma clone expressing CD38; R9: immature myeloid clone expressing CD117. FACS, fluorescence-activated cell sorting.

7 cases reported here were all MDS-EB-1 coexisting with MGUS/MM, therefore, the characteristics of this subtype were the focus of the present study.

The efficiency of treatment for MDS is currently considered unsatisfactory. The median OS of the patients diagnosed with MM coexisting with MDS-EB was 10 months, shorter than that of those with MM coexisting with non-EB (median OS, 19 months). The median OS of the patients diagnosed with MGUS coexisting with MDS$\mathrm{EB}$ was 5 months, while the MGUS/MDS-non-EB group had the longest median OS, at 52 months. An analysis of different subgroups showed that the MGUS/MM coexisting with MDS-EB patients reported in this paper had the least favorable prognosis among all participants (Figure 2).

\section{Discussion}

The coexistence of dual hematological neoplasms is uncommon in clinical scenarios, with a prevalence of $0.3-1.1 \%(25)$. It is an uncommon clinical situation with complex features and complicated treatment, for which the prognosis is unclear. There have been sporadic reports of the simultaneous appearance of primary MDS and MM in patients without prior exposure to chemotherapy.

The presence of 2 clones or 1 disease secondary to the other remains one of the pressing issues to be addressed. Mori et al. considered that the myelodysplastic changes seen in MM could be attributed to MM itself rather than the coexistence of MDS and MM (26). The pathogenesis involves a common stem cell which differentiates into multiple lineage cells. The simultaneous occurrence of AML and MM has been reported, and it was suggested that a common leukemic progenitor provided myeloma as well as leukemia cells, while the mechanism of "lineage infidelity" plays an important role in the development of their "bilinear" differentiation (27). With the development 

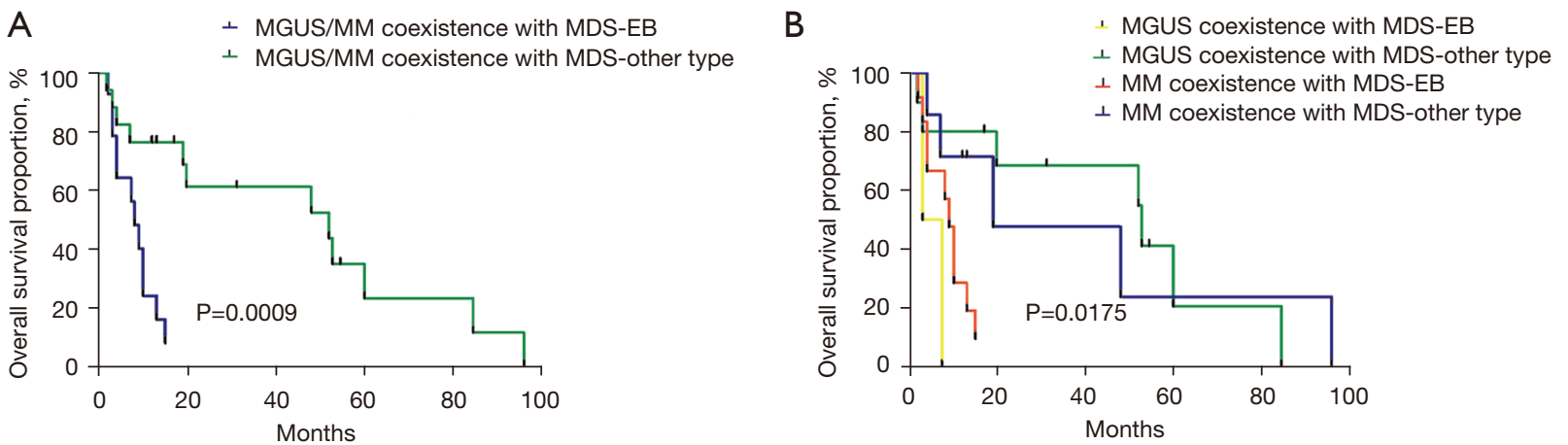

Figure 2 OS of the patients with MGUS/MM and MDS in the subgroup analysis. (A) OS in the MM and MGUS patients with MDS, the median OS of the patients with MGUS/MM and MDS-EB is 8 months, lower than the non-EB group which median OS is 52 months ( $\mathrm{P}=0.0009)$. (B) OS in the MGUS/MM patients with MDS, the median OS of the patients with MGUS/MM and MDS-EB is significantly lower than the non-EB group ( $\mathrm{P}=0.0175)$. OS, overall survival; MGUS/MM, monoclonal gammopathy of underdetermined significance/ multiple myeloma; MDS, myelodysplastic syndrome; MDS-EB, myelodysplastic syndrome with excess blasts.

of technologies such as cytogenetics and next-generation sequencing, researchers are skeptical of the stance that the origin lies in a common stem cell compartment. Using interphase-fluorescence in situ hybridization (FISH; I-FISH) and fluorescence immunophenotype and interphase cytogenetics (FICTION) techniques on bone marrow from a patient with SMM, Quintini et al. reported 1 patient with 2 different clones of MDS and SMM (18). Plasma cells carried only the $t(4 ; 14)$ and the monosomy 13 , while the other bone marrow cells bore isolated $5 \mathrm{q}-$. The clonal independence of MDS and SMM was also supported by the persistence of plasma cells bearing the myeloma genetic changes at the time of disappearance of $5 \mathrm{q}-$, after treatment of AML. Moreover, Zagaria et al. presented a case of a patient diagnosed with MDS 5q- following MGUS (17). In addition to the aberration of chromosome 5, a MYD 88 gene mutation was detected at the time of MDS diagnosis as well at the time point of initial MGUS diagnosis in which no sign of MDS was presented. In addition, FISH analysis on $\mathrm{CD}_{34}{ }^{+}$peripheral stem cells identified the $5 \mathrm{q}-$, but allelespecific polymerase chain reaction (PCR) was negative for the MYD 88 gene mutation in this cell fraction. It was evident that both diseases were independent clones. Nanaji et al. detected 2 cytogenetically distinct clones in the bone marrow of a patient with concurrent MDS and MM (16), of which 1 was hypodiploid with loss of 2 chromosomes and 5 structural chromosome abnormalities, and the second was hyperdiploid with a gain of 6 chromosomes and 3 structural chromosome abnormalities. Wholegenome single-nucleotide polymorphism (SNP) genotyping microarray also showed abnormalities in 2 distinct groups at the mosaicism level. There were 2 gains and 20 losses, including loss of $5 \mathrm{q}$, present approximately $65 \%$ at the mosaicism level and likely corresponded to the MDS clone. A gain of 6 chromosomes was present approximately $10 \%$ at the mosaicism level and likely corresponded to the MM clone. Klimkowska et al. (20) described a cohort of 27 consecutive patients with co-occurring MDS and MM ( $\mathrm{n}=6)$, MGUS ( $\mathrm{n}=20)$, or plasmacytoma $(\mathrm{n}=1)$, and in 21 patients, MDS and plasma-cell disorders (PCDs) were diagnosed simultaneously. To identify potential common founder clones, they performed whole exome sequencing on isolated bone marrow myeloid, plasma, and $\mathrm{T}$ cells from 9 patients. A common founder mutation was absent in all patients, which predisposes the emergence of the 2 diseases to separate clones. From the current research, a growing body of evidence supports the 2 independent clones in the coexistence of MDS with MGUS/MM, and it may be a new type of disease with specific clinical and biological characteristics.

The MDS-EB subtype of MDS is characteristically more like a myeloid clone and has worse prognosis compared to other subtypes. The 7 patients reported in the present study had 2 unrelated clones detected by flow cytometry from bone marrow and blood, where 1 was a malignant myeloid immunophenotype with $\mathrm{CD} 34^{+}$, $\mathrm{CD}_{117^{+}}, \mathrm{HLA}-\mathrm{DR}^{+}$, and $\mathrm{CD} 33^{+}$and the other an abnormal myeloma immunophenotype with $\mathrm{CD} 38^{+}$and $\mathrm{CD} 138^{+}$. By using flow cytometry, Paiva et al. also revealed the presence of MDS-like phenotypic abnormalities (MDS- 
PA) in bone marrow neutrophil, monocytic, and erythroid lineages (28). They investigated 312 newly diagnosed MM patients and eventually up to $33 / 312(11 \%)$ patients showed MDS-PA at diagnosis. However, these 33 patients were not morphologically diagnosed with MDS, and no myeloid blasts were detected. This suggests that the MDS-PA and malignant myeloid clone could be a potential presence in some special MM patients. Notably, as compared to the overall MM population, patients with MDS-PA had significantly inferior progression-free survival (median of $24 v s .37$ months; $\mathrm{P}=0.006$ ) and OS (median of $47 v s$. 73 months; $\mathrm{P}=0.01$ ). The median OS of $\mathrm{MM}$ coexisting with MDS-EB was the shortest ( 9 months). This suggests that the MM clone coexisting with a myeloid clone has great potential as a new poor prognosis factor. Our understanding of the factors that influence prognosis in myeloma has advanced considerably, so the contribution of a range of features can be recognized, including patient fitness, disease biology, genetic lesions, radiological features, and the depth of response, including minimal residual disease (MRD) analysis. However, few people pay attention to the effect of myeloid clone on the prognosis of MM. According to the data of the present study, it should be considered as one of the high-risk factors in the future.

Patients with MGUS/MM and coexisting MDS-EB have shown chemoresistance, and there is currently no successful treatment for these patients. The combination of PI, IMiD, and monoclonal antibody merits more aggressive adaptation, where even initiating early allogeneic stem cell transplantation may be required to improve OS.

The main limitation of this study was that it retrospectively observed a small number of patients, which makes it difficult to comprehensively describe the clinical and prognostic features of the disease. Therefore, longterm observational studies with larger numbers of patients are needed to clarify the clinical course.

\section{Conclusions}

Lineage-specific experiments in the current literature favor the architecture of concomitant "MDS-MM states". However, the precise mechanism of extremely rare cases remains elusive. The patients with coexisting MGUS/ $M M$ and MDS-EB have shown chemoresistance and extremely poor prognosis, which necessitates a need to explore effective therapy to improve the prognosis of patients with the occurrence of such multiple simultaneous malignancies.

\section{Acknowledgments}

We would like to express our immense gratitude to all the physicians and institutions involved in this study for their support. We would like to thank the pathology and hematology departments of the 3 institutions for providing us with patient data. Thanks to the Natural Science Foundation of Liaoning Province for its support.

Funding: This study was supported by the Natural Science Foundation of Liaoning Province (201800875, 20180551257).

\section{Footnote}

Reporting Checklist: The authors have completed the STROBE reporting checklist. Available at https://dx.doi. org/10.21037/apm-21-3210

Data Sharing Statement: Available at https://dx.doi. org/10.21037/apm-21-3210

Conflicts of Interest: All authors have completed the ICMJE uniform disclosure form (available at https://dx.doi. org/10.21037/apm-21-3210). Dr. WY reports that this study was supported by the Natural Science Foundation of Liaoning Province (201800875). Dr. HHW reports that this study was supported by the Natural Science Foundation of Liaoning Province (20180551257). The other authors have no conflicts of interest to declare.

Ethical Statement: The authors are accountable for all aspects of the work in ensuring that questions related to the accuracy or integrity of any part of the work are appropriately investigated and resolved. All procedures performed in this study involving human participants were in accordance with the Declaration of Helsinki (as revised in 2013). The study was approved by The Human Investigation Committee (IRB) of China Medical University (No.:2020PS510K) and informed consent was taken from all the patients.

Open Access Statement: This is an Open Access article distributed in accordance with the Creative Commons Attribution-NonCommercial-NoDerivs 4.0 International License (CC BY-NC-ND 4.0), which permits the noncommercial replication and distribution of the article with the strict proviso that no changes or edits are made and the original work is properly cited (including links to both the 
formal publication through the relevant DOI and the license). See: https://creativecommons.org/licenses/by-nc-nd/4.0/.

\section{References}

1. Hellström-Lindberg E, Tobiasson M, Greenberg P. Myelodysplastic syndromes: moving towards personalized management. Haematologica 2020;105:1765-79.

2. Joseph NS, Gentili S, Kaufman JL, et al. High-risk Multiple Myeloma: Definition and Management. Clin Lymphoma Myeloma Leuk 2017;17S:S80-7.

3. Mailankody S, Pfeiffer RM, Kristinsson SY, et al. Risk of acute myeloid leukemia and myelodysplastic syndromes after multiple myeloma and its precursor disease (MGUS). Blood 2011;118:4086-92.

4. Monaghan SA, Dai L, Mapara MY, et al. Longitudinal bone marrow evaluations for myelodysplasia in patients with myeloma before and after treatment with lenalidomide. Leuk Lymphoma 2013;54:1965-74.

5. Pemmaraju N, Shah D, Kantarjian H, et al. Characteristics and outcomes of patients with multiple myeloma (MM) who develop therapy $(\mathrm{t})$-related myelodysplastic syndrome (MDS), t-chronic myelomonocytic leukemia (CMML), or t-acute myeloid leukemia (AML). Clin Lymphoma Myeloma Leuk 2015;15:110-4.

6. Roeker LE, Larson DR, Kyle RA, et al. Risk of acute leukemia and myelodysplastic syndromes in patients with monoclonal gammopathy of undetermined significance (MGUS): a population-based study of 17315 patients. Leukemia 2013;27:1391-3.

7. Mufti GJ, Hamblin TJ, Clein GP, et al. Coexistent myelodysplasia and plasma cell neoplasia. Br J Haematol 1983;54:91-6.

8. Hamblin TJ. Immunological abnormalities in myelodysplastic syndromes. Semin Hematol 1996;33:150-62.

9. Sato S, Nagai T, Nishikiori Y, et al. Coexistence of myeloma and primary myelodysplastic syndrome (MDS). Rinsho Ketsueki 1992;33:58-62.

10. Florensa L, Vallespí T, Woessner S, et al. Incidence and characteristics of lymphoid malignancies in untreated myelodysplastic syndromes. Leuk Lymphoma 1996;23:609-12.

11. Rios R, Sole F, Gascon F. Simultaneous occurrence of the $5 \mathrm{q}-$ syndrome and multiple myeloma. Clin Lab Haematol 2000;22:49-53.

12. Ortega M, Mallo M, Solé F, et al. 5q- syndrome and multiple myeloma diagnosed simultaneously and successful treated with lenalidomide. Leuk Res 2013;37:1248-50.

13. Li HZ. Report of one case of monoclonal gammopathy with myelodysplastic syndrome and review of the literature. Inner Mongolia Med J 2014;46:252-4.

14. Yoshida Y, Oguma S, Ohno H, et al. Co-occurrence of monoclonal gammopathy and myelodysplasia: a retrospective study of fourteen cases. Int $\mathrm{J}$ Hematol 2014;99:721-5.

15. Gu ZH, Xie X, Mao JJ, et al. Rapid evolving into acute myeloid leukemia in a patient with multiple myeloma and concurrent myelodysplasia after VTD therapy. Int J Clin Exp Med 2015;8:10105-8.

16. Nanaji NM, Chen QC, Singh Z, et al. Concomitant bone marrow involvement by plasma cell myeloma and primary myelodysplastic syndrome with biclonal complex chromosome anomalies. Leuk Lymphoma 2015;56:2470-2.

17. Zagaria A, Coccaro N, Tota G, et al. Myelodysplastic syndrome with 5q deletion following IgM monoclonal gammopathy, showing gene mutation MYD88 L265P. Blood Cells Mol Dis 2015;54:51-2.

18. Quintini M, Arniani S, Ascani S, et al. Identification of two independent clones underlying the co-existence of myelodysplastic syndrome with excess of blasts type 2 and isolated $5 \mathrm{q}-$ and smouldering multiple myeloma. Cytopathology 2020;31:59-62.

19. Nolte F, Mossner M, Jann JC, et al. Concomitant MDS with isolated 5q deletion and MGUS: case report and review of molecular aspects. Eur J Haematol 2017;98:302-10.

20. Klimkowska M, Nannya Y, Gran C, et al. Absence of a common founder mutation in patients with cooccurring myelodysplastic syndrome and plasma cell disorder. Blood 2021;137:1260-3.

21. Rajkumar SV, Dimopoulos MA, Palumbo A, et al. International Myeloma Working Group updated criteria for the diagnosis of multiple myeloma. Lancet Oncol 2014;15:e538-48.

22. Arber DA, Orazi A, Hasserjian R, et al. The 2016 revision to the World Health Organization classification of myeloid neoplasms and acute leukemia. Blood 2016;127:2391-405.

23. Gerecke C, Fuhrmann S, Strifler S, et al. The Diagnosis and Treatment of Multiple Myeloma. Dtsch Arztebl Int 2016;113:470-6.

24. Terpos E, Morgan G, Dimopoulos MA, et al. International Myeloma Working Group recommendations for the treatment of multiple myeloma-related bone disease. J 
Clin Oncol 2013;31:2347-57.

25. Bouchla A, Thomopoulos T, Papageorgiou S, et al. Coexistence of Myeloid and Lymphoid Neoplasms: A Single-Center Experience. Adv Hematol 2019;2019:1486476.

26. Mori M, Abe T, Takei T, et al. The significance of myelodysplasia in untreated patients with multiple myeloma. Rinsho Ketsueki 1992;33:662-5.

27. Akashi K, Harada M, Shibuya T, et al. Simultaneous occurrence of myelomonocytic leukemia and multiple

Cite this article as: Yan W, Qu XY, Li H, Li YC, Li Y, Yao K, Yang W, Wang HH. Coexistence of plasma cell neoplasia and myelodysplastic syndrome with excess blasts: case reports and literature review. Ann Palliat Med 2021;10(12):12431-12440. doi: 10.21037/apm-21-3210 myeloma: involvement of common leukemic progenitors and their developmental abnormality of "lineage infidelity". J Cell Physiol 1991;148:446-56.

28. Paiva B, Puig N, Cedena MT, et al. The presence of MDSlike phenotypic abnormalities (MDS-PA) identifies newly diagnosed multiple myeloma (MM) patients with MDS/ AML-related somatic mutations and inferior survival. Blood 2016;128:375.

(English Language Editor: J. Jones) 